\title{
Lipoprotein(a) is associated with left ventricular systolic dysfunction in a Chinese population of patients with hypertension and without coronary artery disease
}

\author{
Yong Wang ${ }^{1}$, Heng $\mathrm{Ma}^{2}$, Jun Yang ${ }^{2}$, Qiujing Chen ${ }^{3}$, Lin Lu ${ }^{1}$, Ruiyan Zhang ${ }^{1}$
}

\author{
${ }^{1}$ Department of Cardiology, Rui Jin Hospital, Shanghai Jiao Tong University School \\ of Medicine, Shanghai, China \\ ${ }^{2} Y$ Yuhuangding Hospital, Qingdao University School of Medicine, Yantai, China \\ ${ }^{3}$ Institute of Cardiovascular Diseases, Shanghai Jiao Tong University School \\ of Medicine, Shanghai, China
}

Submitted: 25 December 2015

Accepted: 13 April 2016

Arch Med Sci 2017; 13, 5: 1078-1085

DOI: $10.5114 /$ aoms.2016.59875

Copyright @ 2016 Termedia \& Banach

\section{Abstract}

Introduction: Data on relationship between lipoprotein(a) $(\operatorname{Lp}(\mathrm{a})$ ) and nonischemic heart dysfunction are limited. This study is aimed to assess the association between $\mathrm{L} p(\mathrm{a})$ and left ventricular systolic dysfunction in a Chinese population of patients with hypertension and without coronary artery disease (CAD).

Material and methods: This cross-sectional study included 1611 patients with hypertension and without CAD in China. The factors associated with left ventricular ejection fraction (LVEF) were evaluated using univariate and multivariate analysis.

Results: A higher percentage of hypertensive patients with LVEF $<50 \%$ were men, and had lower plasma high-density lipoprotein cholesterol, but higher plasma $L p(a)$, serum creatinine, and hemoglobin levels than those with LVEF $\geq 50 \%$ using univariate analysis. When participants were classified as four groups according to Lp(a) quartiles, LVEF was decreased with increased Lp(a) levels. The prevalence of LVEF $<50 \%$ was increased with Lp(a) quartiles. Multiple linear regression analysis indicated that plasma Lp(a) levels, man, and serum creatinine levels were independently correlated with LVEF in hypertensive patients. Multiple logistic regression analysis indicated that plasma $\mathrm{Lp}(\mathrm{a})$ levels $(\mathrm{OR}=5.566,95 \% \mathrm{Cl}: 1.745-17.758, p=0.004)$ or $\mathrm{Lp}(\mathrm{a})$ quartiles (quartile 4: $\mathrm{OR}=3.234,95 \% \mathrm{Cl}: 1.290-8.105$, quartile 1 as reference, $p=0.012$ ) was independently correlated with LVEF $<50 \%$ with adjustment for other potential confounders. Ordinal logistic regression analysis demonstrated that $\mathrm{LP}(\mathrm{a})(\mathrm{OR}=5.760,95 \% \mathrm{Cl}: 1.831-18.120, p=0.003)$ was independently correlated with different LVEF categories $(\geq 50 \%, 35-49 \%$, and $<35 \%)$ in hypertensive patients.

Conclusions: Left ventricular ejection fraction is decreased with increased plasma Lp(a) levels. Lipoprotein(a) is independently correlated with left ventricular systolic dysfunction in patients with hypertension and without CAD.

Key words: hypertension, coronary artery disease, lipoprotein(a), left ventricular systolic dysfunction.

\section{Introduction}

There is mounting evidence that elevated plasma lipoprotein(a) [Lp(a)] levels contribute significantly to the incidence of cardiovascular diseas-

\author{
Corresponding author: \\ Ruiyan Zhang \\ Department of Cardiology \\ Rui Jin Hospital \\ Shanghai Jiao Tong University \\ School of Medicine \\ 197 Rui Jin $2^{\text {nd }}$ Road \\ 200025 Shanghai, China \\ Phone: +86 2134187554 \\ E-mail: zhangruiyanrj@126. \\ com
}


es (CVDs) including coronary artery disease (CAD) [1-3]. Lipoprotein(a) exhibits strong atherogenic properties and can cause more severe and diffused coronary artery lesions [4]. Numerous studies demonstrate that elevated plasma Lp(a) levels can increase the risk of myocardial infarction (MI) and mortality in CAD patients [5]. Elevated plasma Lp(a) levels are also associated with impaired heart function in CAD and MI patients. Patients with high $L p(a)$ have worse regional wall motion and decreased left ventricular ejection fraction (LVEF) [6]. However, data on the relationship between $\operatorname{Lp}(\mathrm{a})$ and non-ischemic heart dysfunction are limited to date.

Hypertension remains a significant risk factor for development of heart failure, with various mechanisms contributing to both systolic and diastolic dysfunction. The pathogenesis of heart dysfunction includes left ventricular hypertrophy and fibrosis, increased arterial stiffness, activation of the sympathetic nervous system and renin-angiotensin system, etc [7, 8]. Increased arterial stiffness will possibly increase cardiac afterload and further risk of heart failure [8]. Elevated plasma $\mathrm{Lp}(\mathrm{a})$ levels are commonly detected in hypertensive patients and are correlated with target organ damage such as CAD, stroke, and renal failure [9]. The atherogenic effect of high $L p(a)$ levels may possibly also lead to aortic arteriosclerosis and increased arterial stiffness in hypertensive patients. Therefore high $L p(a)$ levels are speculated to be possibly associated with cardiac dysfunction. However, whether $\operatorname{Lp}(\mathrm{a})$ is associated with heart systolic dysfunction in hypertensive patients without CAD remains to be elucidated. Thus, we present this study aimed to assess the association between $\mathrm{Lp}(\mathrm{a})$ and left ventricular systolic dysfunction in a Chinese population of patients with hypertension and without CAD.

\section{Material and methods}

\section{Study subjects}

It was a cross-sectional study which focused on patients with hypertension and without CAD in China. All the participants $(n=1797)$ aged over 20 years old had been consecutively hospitalized in the cardiology or hypertension division of Shanghai Rui Jin Hospital between January and December 2014. Hypertension was diagnosed when systolic blood pressure $\geq 140 \mathrm{~mm} \mathrm{Hg}$, or diastolic blood pressure $\geq 90 \mathrm{~mm} \mathrm{Hg}$, or when actively treated with anti-hypertension drugs. The hypertensive participants in this study were hospitalized to perform clinical assessment and also evaluate whether cardiovascular risk factors, target organ damage or accompanying cardiovascular diseases were present. All participants who had ever received coronary angiography (CAG) or coronary computed tomography (CT) angiography and CAD patients were excluded from this study. Coronary artery disease was diagnosed when diameter stenosis was $>50 \%$ in at least one main coronary artery, or when there was a history of confirmed acute or old myocardial infarction, or a history of revascularization by percutaneous coronary intervention $(\mathrm{PCl})$ or coronary artery bypass graft (CABG). Other exclusion criteria included congenital heart disease, acute infectious diseases, liver failure, hypothyroidism, pregnancy, mental disorder, or cancer. After the subjects with exclusion criteria or incomplete data were removed, there were 1611 participants left.

The study complied with the Declaration of Helsinki. It was also approved by the ethics committee of Shanghai Jiao Tong University and informed consent was obtained from all the participants prior to enrollment.

\section{Blood sampling and laboratory test}

The blood samples were collected from each patient on the next day after admission. Plasma $\mathrm{Lp}(\mathrm{a})$ levels were measured using an immunoturbidimetric assay (Wako Chemicals USA, Inc. Richmond, VA) with a Hitachi 912 autoanalyzer (Roche Diagnostics, Basel, Switzerland). Meanwhile, the levels of plasma total cholesterol, total triglyceride, high-density lipoprotein cholesterol (HDL-C), low-density lipoprotein cholesterol (LDL-C), plasma glucose, and serum creatinine were analyzed by the automatic biochemical analyzer. Blood cell tests were executed using an automated blood cell counter (Beckman Coulter LH750, CA, USA). Laboratory test results were generated by personnel blinded to the clinical characteristics of the study participants.

\section{Blood pressure and echocardiographic measurements}

Blood pressure was measured using a standardized automatic electronic sphygmomanometer (HEM-741C; Omron, Tokyo, Japan). One experienced physician performed 3 blood pressure measurements using an American Heart Association protocol after study participants had at least a 5 min rest in the sitting position. Study participants avoided eating, drinking alcohol, drinking coffee, smoking, exercising, and bathing for $30 \mathrm{~min}$ before taking these measurements. The mean of 3 blood pressure values was calculated and used for further analysis.

One echocardiographer blinded to the biochemical examination results of the study participants performed all echocardiographic measurements using the Phillips IE33 device according to 
the American Society of Echocardiography (ASE) recommendations. M-mode, two-dimensional, and color Doppler images were first recorded, and then analyzed offline. Left ventricular ejection fraction assessment was based on two-dimensional echocardiography using the quantitative two-dimensional biplane volumetric Simpson method from 4- and 2-chamber views. Intraobserver reproducibility was assessed among 25 randomly selected patients. No significant difference was found (intra-observer: mean difference: $1.4 \pm 0.20 \%, p=0.701$ ).

\section{Clinical data collection}

A case report form was developed to assess the general characteristics, clinical diagnosis, medical history, medical treatment, and biochemical examination. Blood pressure measurement recorded in the form was obtained on admission. Current smoking was determined when subjects were smoking currently and had smoked more than one cigarette daily for at least one year continuously. Diabetes mellitus was diagnosed by a fasting plasma glucose test showing $\geq 7.0 \mathrm{mmol} / \mathrm{l}$, or by a random plasma glucose test showing $\geq 11.1$ $\mathrm{mmol} / \mathrm{l}$, or when they were actively receiving therapy using insulin or oral medications for diabetes. Medical therapy including statins, angiotensin converting enzyme inhibitors (ACEI), angiotensin receptor antagonists (ARB), calcium antagonists, and $\beta$-blockers was also included in the case report form.

\section{Statistical analysis}

Data entry and management were performed on Epidata software, version 3.1 (Epidata Association, Odense, Denmark). Data were analyzed using the software program SPSS 13.0 (SPSS Inc., Chicago, IL, USA). Continuous variables were expressed as the mean \pm standard deviation, and categorical variables as a percentage. The $\chi^{2}$ test and trend test were used to compare categorical variables among several groups. The independent-sample $t$-test was used to compare continuous variables between two groups. The trend test was used to compare continuous variables among more than two groups. Multiple linear regression analysis which included variables identified as statistically significant in the univariate analysis was used to assess the independence of the association between LVEF and Lp(a). Multiple logistic regression analysis was used to assess the independence of the association between LVEF $<50 \%$ and Lp(a). Ordinal logistic regression analysis was used to assess the association between different LVEF categories and $L p(a)$. Two-sided $p<0.05$ was considered significant.

\section{Results}

\section{Study population characteristics}

In total, 1611 participants with hypertension and without CAD were included in the final statistical analysis. General characteristics of these participants are shown in Table I. Their mean age was $62.4 \pm 9.67$ years. Eight hundred and two (49.8\%) participants were men. A total of 1300 study subjects $(80.7 \%)$ had received antihypertensive drugs. Furthermore, 506 (31.4\%) subjects, 970 (60.2\%) subjects, and 330 subjects (20.5\%) were treated with $\beta$-blockers, ACEI or ARB, and calcium antagonists, respectively. The average LVEF was 65.3 $\pm 7.09 \%$. The prevalence of LVEF $<50 \%$ was $3.6 \%$. A higher percentage of hypertensive patients with LVEF $<50 \%$ were men than those with LVEF $\geq 50 \%$ $(p<0.001)$. The hypertensive patients with LVEF $<50 \%$ had lower HDL-C levels, but higher plasma Lp(a), serum creatinine, and hemoglobin levels than those with LVEF $\geq 50 \%$ (all $p<0.05$ ).

\section{Factors associated with plasma $\operatorname{Lp}(\mathrm{a})$ levels}

We also explored the factors associated with plasma Lp(a) levels using univariate and multivariate analysis. The data indicated that hypertensive patients with $\mathrm{Lp}(\mathrm{a})>0.13 \mathrm{~g} / \mathrm{l}$ (median value) had higher LDL-C levels (2.62 $\pm 0.80 \mathrm{mmol} / \mathrm{l}, n=774$, vs. $2.44 \pm 0.78 \mathrm{mmol} / \mathrm{l}, n=837, p<0.001)$, plasma total cholesterol levels $(4.35 \pm 0.96 \mathrm{mmol} / \mathrm{l}$, $n=774$, vs. $4.16 \pm 0.95 \mathrm{mmol} / \mathrm{l}, n=837, p<0.001)$, but lower LVEF $(64.7 \pm 7.78, n=774$, vs. $65.9 \pm 6.33$, $n=837, p<0.001)$ than those with $\operatorname{Lp}(\mathrm{a}) \leq 0.13 \mathrm{~g} / \mathrm{l}$ in the univariate analysis. Furthermore, independent factors associated with higher $\mathrm{Lp}(\mathrm{a})$ (> $0.13 \mathrm{~g} / \mathrm{l}$ ) were evaluated using multiple logistic regression analysis. Variables identified as statistically significant in the univariate analysis and other important demographic characteristics including age, sex, and diabetes mellitus entered in the regression equation. The results showed that LDL-C $(\mathrm{OR}=1.440,95 \% \mathrm{Cl}: 1.040-1.994$, $p=0.028)$ and LVEF (OR $=0.971,95 \% \mathrm{Cl}: 0.957-$ $0.986, p<0.001)$ were independent factors correlated with higher $L p(a)$ in patients with hypertension and without CAD.

\section{Association between LVEF and Lp(a)}

The participants were classified into four groups according to plasma $L p(a)$ levels. The $L p(a)$ ranges in quartile 1 , quartile 2 , quartile 3 , and quartile 4 were < $0.07 \mathrm{~g} / \mathrm{l}, 0.07-0.13 \mathrm{~g} / \mathrm{l}, 0.14-0.24 \mathrm{~g} / \mathrm{l}$, and $>0.24 \mathrm{~g} / \mathrm{l}$, respectively. The LVEF decreased with increasing plasma Lp(a) levels $(66.3 \pm 6.19 \%$ vs. $65.7 \pm 6.43 \%$ vs. $64.8 \pm 7.30 \%$ vs. $64.5 \pm 8.21 \%$, $p$ for trend $=0.002$, Figure $1 \mathrm{~A})$. At the same time, the prevalence of LVEF $<50 \%$ increased with $L p(a)$ 
Table I. Clinical characteristics of hypertensive patients with LVEF $\geq 50 \%$ or $<50 \%$

\begin{tabular}{|c|c|c|c|c|}
\hline Variables & $\begin{array}{c}\text { All } \\
(n=1611)\end{array}$ & $\begin{array}{l}\mathrm{EF} \geq 50 \% \\
(n=1553)\end{array}$ & $\begin{array}{c}\mathrm{EF}<50 \% \\
(n=58)\end{array}$ & $P$-value \\
\hline Age [years] & $62.4 \pm 9.67$ & $62.5 \pm 9.65$ & $60.4 \pm 10.0$ & 0.103 \\
\hline Men, $n(\%)$ & $802(49.8)$ & $755(48.6)$ & $47(81.0)$ & $<0.001$ \\
\hline Current smoking, $n(\%)$ & $249(15.5)$ & $238(15.3)$ & $11(19.0)$ & 0.451 \\
\hline Diabetes mellitus, $n(\%)$ & $391(24.3)$ & $378(24.3)$ & $13(22.4)$ & 0.737 \\
\hline BMI $\left[\mathrm{kg} / \mathrm{m}^{2}\right]$ & $25.3 \pm 3.34$ & $25.2 \pm 3.32$ & $25.6 \pm 3.55$ & 0.469 \\
\hline $\mathrm{SBP}[\mathrm{mm} \mathrm{Hg}]$ & $139 \pm 19.0$ & $139 \pm 18.7$ & $137 \pm 22.8$ & 0.278 \\
\hline $\mathrm{DBP}[\mathrm{mm} \mathrm{Hg}]$ & $77.0 \pm 12.1$ & $77.0 \pm 11.9$ & $76.2 \pm 14.3$ & 0.340 \\
\hline Total cholesterol [mmol/l] & $4.25 \pm 0.96$ & $4.26 \pm 0.96$ & $4.12 \pm 1.00$ & 0.274 \\
\hline Total triglyceride [mmol/l] & $1.73 \pm 1.04$ & $1.73 \pm 1.02$ & $1.67 \pm 1.55$ & 0.663 \\
\hline LDL-C [mmol/l] & $2.53 \pm 0.80$ & $2.53 \pm 0.79$ & $2.50 \pm 0.84$ & 0.781 \\
\hline $\mathrm{HDL}-\mathrm{C}[\mathrm{mmol} / \mathrm{l}]$ & $1.18 \pm 0.31$ & $1.18 \pm 0.31$ & $1.09 \pm 0.34$ & 0.034 \\
\hline $\operatorname{Lp}(\mathrm{a})[g / l]$ & $0.19 \pm 0.18$ & $0.19 \pm 0.18$ & $0.25 \pm 0.20$ & 0.016 \\
\hline $\mathrm{HbA}_{1 \mathrm{c}}(\%)$ & $6.13 \pm 1.02$ & $6.12 \pm 1.03$ & $6.31 \pm 0.98$ & 0.232 \\
\hline Fasting plasma glucose [mmol/l] & $5.27 \pm 1.28$ & $5.26 \pm 1.26$ & $5.30 \pm 1.75$ & 0.838 \\
\hline $2 \mathrm{~h}$ plasma glucose $[\mathrm{mmol} / \mathrm{l}]$ & $8.22 \pm 3.27$ & $8.21 \pm 3.30$ & $8.54 \pm 2.38$ & 0.566 \\
\hline Fasting plasma insulin $[\mu \mathrm{lU} / \mathrm{ml}]$ & $11.6 \pm 20.7$ & $11.7 \pm 21.0$ & $8.81 \pm 6.39$ & 0.467 \\
\hline Serum creatinine $[\mu \mathrm{mol} / \mathrm{l}]$ & $75.7 \pm 34.7$ & $75.0 \pm 34.6$ & $94.5 \pm 32.0$ & $<0.001$ \\
\hline Hemoglobin $[\mathrm{g} / \mathrm{l}]$ & $134 \pm 15.2$ & $133 \pm 15.1$ & $140 \pm 18.5$ & 0.003 \\
\hline LVEF (\%) & $65.3 \pm 7.09$ & $66.3 \pm 4.79$ & $38.7 \pm 7.16$ & $<0.001$ \\
\hline Statin use, $n(\%)$ & $306(19.0)$ & $294(18.9)$ & $12(20.7)$ & 0.737 \\
\hline$\beta$-blockers, $n(\%)$ & $506(31.4)$ & $485(31.2)$ & $21(36.2)$ & 0.423 \\
\hline ACEI or ARB, $n(\%)$ & $970(60.2)$ & $931(59.9)$ & $39(67.2)$ & 0.265 \\
\hline Calcium antagonist, $n(\%)$ & $330(20.5)$ & $322(20.7)$ & $8(13.8)$ & 0.198 \\
\hline
\end{tabular}

$B M I$ - body mass index, SBP - systolic blood pressure, LDL-C - low-density lipoprotein cholesterol, HDL-C - high-density lipoprotein cholesterol, $L p(a)$ - lipoprotein (a), HbA 1 - glycated hemoglobin, LVEF - left ventricular ejection fraction, ACEI - angiotensin-converting enzyme inhibitors, $A R B$ - angiotensin receptor antagonists. Values are means $\pm S D$ or numbers with percentage in parentheses.

quartiles $(2.2 \%$ vs. $2.6 \%$ vs. $4.6 \%$ vs. $5.2 \%, p$ for trend $=0.008$, Figure $1 \mathrm{~B})$. The prevalence of LVEF $<50 \%$ in patients of quartile 4 was increased by 2.4-fold compared with that in patients of quartile 1.

In order to explore the plasma $\operatorname{Lp}(\mathrm{a})$ levels with different severity of heart dysfunction, we divided the participants into three groups according to LVEF ( $\geq 50 \%, 35-49 \%$, and $<35 \%$ ). We found that plasma $L p(a)$ levels were further increased with more severely impaired LVEF $(0.19 \pm 0.18 \mathrm{~g} / \mathrm{l}$ vs. 0.23 $\pm 0.18 \mathrm{~g} /$ l vs. $0.28 \pm 0.22 \mathrm{~g} / \mathrm{l}, p=0.031$, Figure $1 \mathrm{C}$ ).

\section{Independent correlation between $\mathrm{Lp}(\mathrm{a})$ and LVEF}

Multiple linear regression analysis was used to evaluate the independent factors associated with LVEF. The plasma Lp(a) levels entered the regression analysis as a linear variable. Other variables identified as statistically significant in the univariate analysis ( $E F<50 \%$ vs. $E F \geq 50 \%$ ) also entered the regression equation. Age and sex were also included as important demographic characteristics. Furthermore, factors associated with plasma Lp(a) levels also entered the regression equation. Finally, various factors including age, male sex, plasma Lp(a) levels, serum creatinine levels, plasma HDL-C levels, plasma LDL-C levels, and hemoglobin were included in the multiple linear regression analysis. The results indicated that plasma Lp(a) levels, male sex, and serum creatinine levels were independent factors correlated with LVEF in patients with hypertension and without CAD (Table II).

\section{Independent correlation between Lp(a) and LVEF $<50 \%$}

Independent factors associated with LVEF $<50 \%$ were evaluated using multiple logistic regression analysis. The plasma Lp(a) levels entered the logistic regression analysis as a linear variable or as quartiles respectively (model 1 or model 2). Age, sex, and other variables identified as statistically significant in the univariate analysis were also included in the regression equation. The regression analysis in model 1 indicated that plasma Lp(a) levels (OR $=5.566,95 \% \mathrm{Cl}: 1.745-17.758$, $p=0.004)$ and male sex were independent fac- 
A

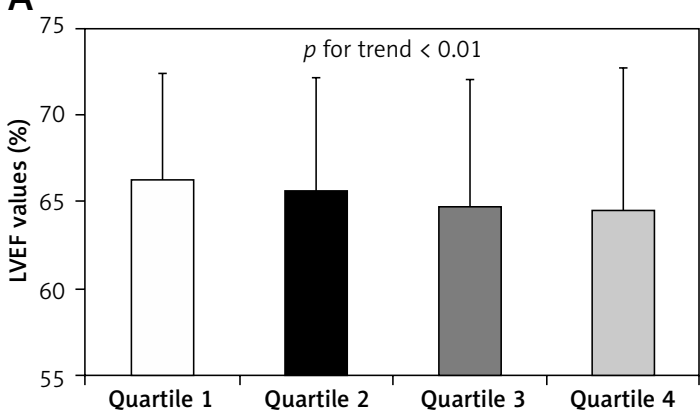

C

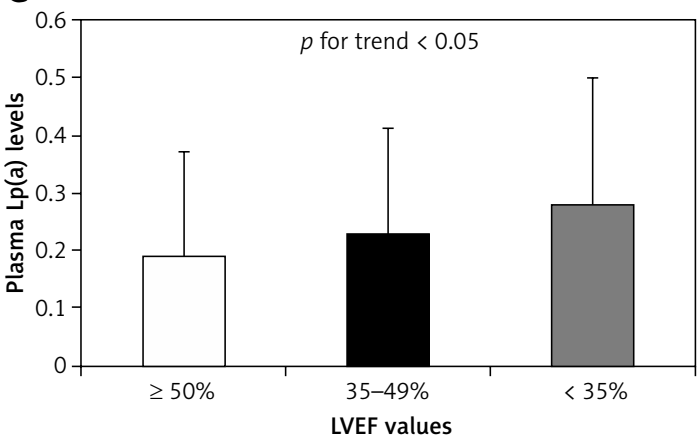

B

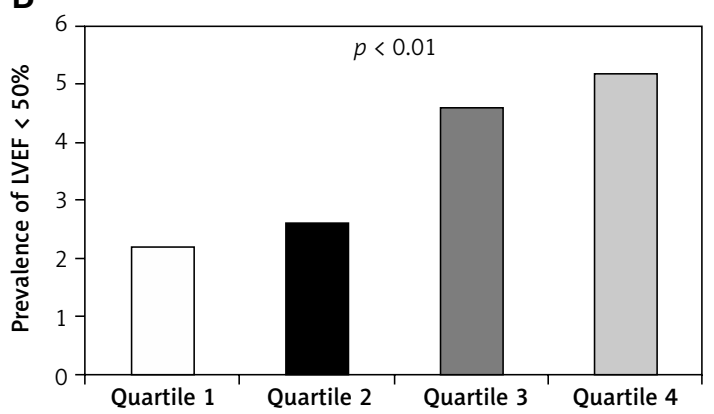

Figure 1. Association between LVEF and Lp(a). A - LVEF values in patients with different $L p(a)$ quartiles. The $L p(a)$ ranges in quartile 1 , quartile 2 , quartile 3 , and quartile 4 were $<0.07 \mathrm{~g} / \mathrm{l}, 0.07-0.13 \mathrm{~g} / \mathrm{l}, 0.14-0.24 \mathrm{~g} / \mathrm{l}$, and >0.24 g/l, respectively. $N=367$ for quartile 1,470 for quartile 2,370 for quartile 3, and 404 for quartile 4. $P$ for trend $<0.01$. B - Prevalence of LVEF $<50 \%$ in patients with different Lp(a) quartiles. $P<0.01$ among four group. $C$ - Plasma $L p(a)$ levels in patients with different LVEF categories $(<35 \%, 35-49 \%$, $\geq 50 \%)$. $N=1553$ for LVEF $<35 \%, 38$ for LVEF of $35-49 \%$, and 20 for $L V E F \geq 50 \%$. $P$ for trend $<0.05$

Table II. Factors associated with LVEF using multiple linear regression analysis

\begin{tabular}{|lcccc|}
\hline Variables & Standard $\beta$ coefficient & $\beta$ coefficient & $\begin{array}{c}\beta \text { coefficient's } \\
95 \% \text { Cl }\end{array}$ & $P$-value \\
\hline Age [years] & 0.034 & 0.025 & -0.012 to 0.062 & 0.188 \\
\hline Male $(1=$ yes, $0=\mathrm{no})$ & -0.116 & -1.616 & -2.473 to -0.759 & $<0.001$ \\
\hline Serum creatinine $[\mu \mathrm{mol} / \mathrm{l}]$ & -0.064 & -0.013 & -0.023 to -0.003 & 0.013 \\
\hline HDL-C $[\mathrm{mmol} / \mathrm{l}]$ & 0.010 & 0.228 & -0.919 to 1.375 & 0.697 \\
\hline LDL-C $[\mathrm{mmol} / \mathrm{l}]$ & 0.018 & 0.160 & -0.278 to 0.599 & 0.474 \\
\hline Hemoglobin $[\mathrm{g} / \mathrm{l}]$ & -0.019 & -0.009 & -0.035 to 0.018 & 0.525 \\
\hline Lp(a) $[\mathrm{g} / \mathrm{l}]$ & -0.081 & -3.106 & -4.970 to -1.242 & 0.001 \\
\hline
\end{tabular}

HDL-C - high-density lipoprotein cholesterol, LDL-C - low-density lipoprotein cholesterol, Lp(a) - lipoprotein(a), HbA ${ }_{1 c}-g$ lycated hemoglobin.

tors correlated with $\mathrm{LVEF}<50 \%$ in hypertensive patients. At the same time, plasma Lp(a) levels (quartile 4: OR $=3.234,95 \% \mathrm{Cl}: 1.290-8.105, p=$ 0.012 , quartile 1 as reference), male sex, and serum creatinine levels were independently correlated with LVEF $<50 \%$ in model 2 (Table III).

\section{Independent correlation between Lp(a) and LVEF categories}

In order to investigate which factors were independently correlated with the severity of left ventricular systolic dysfunction, ordinal logistic regression analysis was used to evaluate the correlation between various factors and different LVEF categories ( $\geq 50 \%, 35-49 \%$, and $<35 \%$ ). Age, male sex, plasma Lp(a) levels, serum creatinine levels, plasma HDL-C levels, plasma LDL-C levels, and hemoglobin (as in the above-described multiple linear regression analysis) were included in the ordinal logistic regression analysis. The results showed that plasma $L p(a)$ levels $(O R=5.760$, 95\% Cl: 1.831-18.120, $p=0.003)$, male sex, and serum creatinine levels were independently correlated with different LVEF categories (Table IV).

\section{Discussion}

The incidence, prevalence of and overall mortality from ischemic heart disease and chronic heart failure (CHF) have been increasing in recent decades [8, 10-13]. Hypertension, alone or in combination with ischemic heart disease, precedes the development of heart failure and causes more deaths. The relative risk of $\mathrm{CHF}$ among patients with hypertension is significantly higher than in the general population [14]. Hypertension is one of the most important modifiable risk fac- 
Table III. Adjusted OR of factors associated with LVEF $<50 \%$ using multiple logistic regression analysis

\begin{tabular}{|c|c|c|c|c|c|}
\hline Variables & OR $(95 \% \mathrm{Cl})$ & $P$-value & Variables & OR $(95 \% \mathrm{Cl})$ & $P$-value \\
\hline \multicolumn{3}{|l|}{ Model 1: } & \multicolumn{3}{|l|}{ Model 2: } \\
\hline Age [years] & $0.993(0.964-1.022)$ & 0.628 & Age [years] & $0.992(0.963-1.022)$ & 0.588 \\
\hline Male $(1=$ yes, $0=$ no $)$ & $3.996(1.800-8.868)$ & 0.001 & Male $(1=$ yes, $0=$ no $)$ & $3.681(1.666-8.135)$ & 0.001 \\
\hline $\begin{array}{l}\text { Serum creatinine } \\
{[\mu \mathrm{mol} / \mathrm{l}]}\end{array}$ & $1.004(1.000-1.008)$ & 0.055 & $\begin{array}{l}\text { Serum creatinine } \\
{[\mu \mathrm{mol} / \mathrm{l}]}\end{array}$ & $1.004(1.000-1.008)$ & 0.036 \\
\hline $\mathrm{HDL}-\mathrm{C}[\mathrm{mmol} / \mathrm{l}]$ & $0.784(0.283-2.170)$ & 0.639 & $\mathrm{HDL}-\mathrm{C}[\mathrm{mmol} / \mathrm{l}]$ & $0.715(0.257-1.990)$ & 0.521 \\
\hline LDL-C [mmol/l] & $0.980(0.689-1.394)$ & 0.910 & LDL-C [mmol/l] & $0.963(0.676-1.371)$ & 0.834 \\
\hline Hemoglobin $[\mathrm{g} / \mathrm{l}]$ & $1.006(0.987-1.026)$ & 0.528 & Hemoglobin $[\mathrm{g} / \mathrm{l}]$ & $1.007(0.988-1.027)$ & 0.468 \\
\hline $\operatorname{Lp}(\mathrm{a})[\mathrm{g} / \mathrm{l}]:$ & $5.566(1.745-17.758)$ & 0.004 & $\operatorname{Lp}(a):$ & - & 0.030 \\
\hline \multicolumn{3}{|l|}{-} & Quartile 1 & \multicolumn{2}{|l|}{ Reference } \\
\hline- & & & Quartile 2 & $1.487(0.560-3.951)$ & 0.426 \\
\hline- & & & Quartile 3 & $2.746(1.083-6.963)$ & 0.033 \\
\hline- & & & Quartile 4 & $3.234(1.290-8.105)$ & 0.012 \\
\hline
\end{tabular}

HDL-C - high-density lipoprotein cholesterol, LDL-C - low-density lipoprotein cholesterol, Lp(a) - lipoprotein(a), HbA - glycated hemoglobin. $L p(a)$ entered the regression equation as a continuous variable $(g / l)$ in model $1 . L p(a)$ quartiles also entered the regression equation as a categorical variable in model 2 (quartile 1 as reference).

tors for CHF [15]. Hypertension and CHF are both major public health problems in China. The prevalence of LVEF $<50 \%$ in Chinese hypertensive patients without CAD is $3.6 \%$ in this study. Thus, heart dysfunction in hypertensive patients should receive more attention.

In this study, a higher percentage of hypertensive patients with LVEF < 50\% were men, and had lower plasma HDL-C levels, but higher plasma Lp(a), serum creatinine, and hemoglobin levels than those with $E F \geq 50 \%$. The data from the Framingham Heart Study also indicated that male sex significantly predicted the new onset of heart failure and reduced ejection fraction (HFREF) during an 8-year follow-up [16]. Chronic kidney disease (CKD) was very common in CHF and was independently associated with longterm adverse outcomes in some cohort studies [17, 18]. Large epidemiological studies revealed that more than $30 \%$ of patients hospitalized for decompensated HF have a glomerular filtration rate $(G F R)<60 \mathrm{ml} / \mathrm{min} / 1.73 \mathrm{~m}^{2}[19,20]$. Numerous studies have confirmed the role of inflammation in both ischemic and non-ischemic heart failure. The HDL-C is anti-inflammatory and may help to improve heart failure. A previous study showed that HDL-C was decreased in patients with advanced heart failure waiting for heart transplant [21]. Framingham Heart Study also suggested that low HDL-C and a high hematocrit can predict incident cardiac failure during long periods of follow-up [22].

In this study, $\operatorname{Lp}(\mathrm{a})$ was one of the significant factors associated with left ventricular dysfunc-
Table IV. Adjusted OR of factors associated with different LVEF levels using ordinal logistic regression analysis

\begin{tabular}{|lcc|}
\hline Variables & OR $(95 \% \mathrm{Cl})$ & $P$-value \\
\hline Age [years] & $0.992(0.964-1.021)$ & 0.603 \\
\hline $\begin{array}{l}\text { Male } \\
(1=\text { yes, } 0=\text { no })\end{array}$ & $4.007(1.808-8.891)$ & 0.001 \\
\hline $\begin{array}{l}\text { Serum creatinine } \\
\text { [ } \mathrm{mol} / \mathrm{l}]\end{array}$ & $1.004(1.000-1.007)$ & 0.030 \\
\hline HDL-C $[\mathrm{mmol} / \mathrm{l}]$ & $0.772(0.279-2.138)$ & 0.618 \\
\hline LDL-C [mmol/l] & $0.984(0.693-1.398)$ & 0.928 \\
\hline Hemoglobin $[\mathrm{g} / \mathrm{l}]$ & $1.006(0.987-1.026)$ & 0.517 \\
\hline Lp (a) $[\mathrm{g} / \mathrm{l}]$ & $5.760(1.831-18.120)$ & 0.003 \\
\hline
\end{tabular}

$H D L-C$ - high-density lipoprotein cholesterol, LDL-C - low-density lipoprotein cholesterol, $\mathrm{Lp}(\mathrm{a})$ - lipoprotein(a), $H b A_{1 c}$ - glycated hemoglobin. $L p(a)$ was included in the regression equation as a continuous variable [g/l] to evaluate the association with different LVEF categories (variable assignment: $\geq 50 \%$ as $0,35-$ $49 \%$ as 1 , and $<35 \%$ as 2 ).

tion. Lp(a) was independently correlated with EF, $\mathrm{EF}<50 \%$, or different EF categories respectively after adjusting for other potential confounders. The $\operatorname{Lp}(a)$ was proved to be strongly associated with CAD and MI [4, 5], which can be promoted by hypertension and can increase the onset of heart failure. Thus, $\operatorname{Lp}(\mathrm{a})$ might be correlated with left ventricular dysfunction in hypertensive patients by promoting the development of ischemic heart disease. However, we excluded CAD and MI patients in this study. It means that the association between $\mathrm{Lp}(\mathrm{a})$ and heart dysfunction in this Chi- 
nese population of hypertensive patients is possibly independent from ischemic heart disease. In fact, a previous study showed that the $L p(a)$ levels in age- and sex-matched normal people were significantly lower than those of either the CHF or the heart transplant recipients, who included both ischemic and non-ischemic heart disease patients [21]. But the sample size was small in that study, and multiple regression analysis was not used to evaluate the independent correlation between $\mathrm{Lp}(\mathrm{a})$ and heart failure. However, we evaluated the independent association between $\operatorname{Lp}(\mathrm{a})$ and non-ischemic heart systolic dysfunction in hypertensive patients in this study. Also, a recently published study demonstrated that elevated Lp(a) levels and corresponding LPA risk genotypes were associated with an increased risk of heart failure consistent with a causal association in a general population. The association appeared to be partly mediated by myocardial infarction and aortic valve stenosis [23, 24]. However, the authors could not exclude an additional contribution from $\operatorname{Lp}(\mathrm{a})$, also acting via other presently unknown mechanisms that affect cardiac function independently of CAD and aortic valve stenosis. They also speculated that high Lp(a) levels may possibly lead to increased arterial stiffness, including vascular noncompliance in the aorta, which will increase afterload, and which had been strongly associated with increased risk of HF. Thus, we speculate that the association between high $\operatorname{Lp}(\mathrm{a})$ and low LVEF was possibly mediated by increased arterial stiffness, aortic valve stenosis, or other unknown mechanisms in our study population.

Our study should also be interpreted within the context of its limitations. First, this is a cross-sectional study, so a causal relationship between $L p(a)$ and heart systolic dysfunction remains to be confirmed. Pathophysiological mechanisms of the association between Lp(a) and LVEF are unknown and need further studies. Second, although the overall population size is reasonable, the sample size of subjects with low LVEF is rather small, which might impair the power. Third, subjects had received hypotensive drugs before blood pressure was measured. Thus, blood pressure in this study cannot be considered as a good index of hypertension severity. It is a limitation that we did not include any measure of the severity of hypertension.

In conclusion, LVEF decreases with increasing plasma $L p(a)$ levels. $L p(a)$ is independently correlated with left ventricular systolic dysfunction in patients with hypertension and without CAD.

\section{Acknowledgments}

Yong Wang and Heng Ma contributed equally to this work.
We are grateful to the subjects who participated in the study, and for the physicians' assistance in this study.

This study was sponsored by the National Natural Science Foundation of China (81500335, 81401385, and 81571636), Shanghai Natural Science Foundation (13ZR1433500), and the 56th Project Funded by the China Postdoctoral Science Foundation (2014M560346).

\section{Conflict of interest}

The authors declare no conflicts of interest.

\section{References}

1. Nordestgaard BG, Chapman MJ, Ray K, et al. Lipoprotein(a) as a cardiovascular risk factor: current status. Eur Heart J 2010; 31: 2844-53.

2. Dube JB, Boffa MB, Hegele RA, Koschinsky ML. Lipoprotein(a): more interesting than ever after 50 years. Curr Opin Lipidol 2012; 23: 133-40.

3. Reiner Z, Catapano AL, De Backer G, et al. ESC/EAS Guidelines for the management of dyslipidaemias: the Task Force for the management of dyslipidaemias of the European Society of Cardiology (ESC) and the European Atherosclerosis Society (EAS). Eur Heart J 2011; 32: 1769-818.

4. Wang XL, Tam C, McCredie RM, Wilcken DE. Determinants of severity of coronary artery disease in Australian men and women. Circulation 1994; 89: 1974-81.

5. Kamstrup PR, Tybjaerg-Hansen A, Steffensen R, Nordestgaard BG. Genetically elevated lipoprotein(a) and increased risk of myocardial infarction. JAMA 2009; 301: 2331-9.

6. Aksoy M, Kepekçi Y, Göktekin O, et al. Relation of plasma lipoprotein(a) with myocardial viability and left ventricular performance in survivors of myocardial infarction. Jpn Heart J 1999; 40: 703-13.

7. Kannan A, Janardhanan R. Hypertension as a risk factor for heart failure. Curr Hypertens Rep 2014; 16: 447.

8. Marti CN, Gheorghiade M, Kalogeropoulos AP, Georgiopoulou VV, Quyyumi AA, Butler J. Endothelial dysfunction, arterial stiffness, and heart failure. J Am Coll Cardiol 2012; 60: 1455-69.

9. Catena C, Novello M, Lapenna R, et al. New risk factors for atherosclerosis in hypertension: focus on the prothrombotic state and lipoprotein(a). J Hypertens 2005; 23: 1617-31.

10. Qi X, Xu M, Yang H, et al. Comparing mortality and myocardial infarction between coronary artery bypass grafting and drug-eluting stenting in patients with diabetes mellitus and multivessel coronary artery disease: a meta-analysis. Arch Med Sci 2014; 10: 411-8.

11. Wang L, Qu P, Zhao J, Chang Y. NLRP3 and downstream cytokine expression elevated in the monocytes of patients with coronary artery disease. Arch Med Sci 2014; 10: 791-800.

12. Dodani S, Dong L, Guirgis FW, Reddy ST. Carotid intima media thickness and low high-density lipoprotein (HDL) in South Asian immigrants: could dysfunctional HDL be the missing link? Arch Med Sci 2014; 10: 870-9.

13. Liszka J, Haberka M, Tabor Z, Finik M, Gąsior Z. Two-dimensional speckle-tracking echocardiography assessment of left ventricular remodeling in patients after 
myocardial infarction and primary reperfusion. Arch Med Sci 2014; 10: 1091-100.

14. Dorobantu M, Tautu OF, Darabont R, et al. Objectives and methodology of Romanian SEPHAR II Survey. Project for comparing the prevalence and control of cardiovascular risk factors in two East-European countries: Romania and Poland. Arch Med Sci 2015; 11: 715-23.

15. He J, Ogden LG, Bazzano LA, Vupputuri S, Loria C, Whelton PK. Risk factors for congestive heart failure in US men and women: NHANESI epidemiologic follow-up study. Arch Intern Med 2001; 161: 996-1002.

16. Meredith PA, Ostergren J. From hypertension to heart failure are there better primary prevention strategies? J Renin Angiotensin Aldosterone Syst 2006; 7: 64-73.

17. Ho JE, Lyass A, Lee DS, et al. Predictors of new-onset heart failure: differences in preserved versus reduced ejection fraction. Circ Heart Fail 2013; 6: 279-86.

18. Hamaguchi S, Tsuchihashi-Makaya M, Kinugawa S, et al. Chronic kidney disease as an independent risk for long-term adverse outcomes in patients hospitalized with heart failure in Japan. Report from the Japanese Cardiac Registry of Heart Failure in Cardiology (JCARE(ARD). Circ J 2009; 73: 1442-7.

19. Zamora E, Lupón J, Vila J, et al. Estimated glomerular filtration rate and prognosis in heart failure: value of the Modification of Diet in Renal Disease Study-4, chronic kidney disease epidemiology collaboration, and Cockroft-Gault formulas. J Am Coll Cardiol 2012; 59: 1709-15.

20. Heywood JT, Fonarow GC, Costanzo MR, et al. High prevalence of renal dysfunction and its impact on outcome in 118,465 patients hospitalized with acute decompensated heart failure: a report from the ADHERE database. J Card Fail 2007; 13: 422-30.

21. Fonarow GC, Abraham WT, Albert NM, et al. Influence of a performance-improvement initiative on quality of care for patients hospitalized with heart failure: results of the Organized Program to Initiate Lifesaving Treatment in Hospitalized Patients With Heart Failure (OPTIMIZE-HF). Arch Intern Med 2007; 167: 1493-502.

22. Cooke GE, Eaton GM, Whitby G, et al. Plasma atherogenic markers in congestive heart failure and posttransplant (heart) patients. J Am Coll Cardiol 2000; 36: 509-16.

23. Kannel WB, Cupples A. Epidemiology and risk profile of cardiac failure. Cardiovasc Drugs Ther 1988; 2 Suppl 1: 387-95.

24. Kamstrup PR, Nordestgaard BG. Elevated lipoprotein(a) levels, LPA risk genotypes, and increased risk of heart failure in the general population. JACC Heart Fail 2016; 4: 78-87. 